Editorial

\title{
Algorithmic Actants in Practice, Theory, and Method
}

\author{
Rodrigo Zamith ${ }^{1, *}$ and Mario Haim ${ }^{2}$ \\ 1 Journalism Department, University of Massachusetts-Amherst, 01002 Amherst, USA; E-Mail: rzamith@umass.edu \\ 2 Department of Communication and Media Studies, University of Leipzig, 04109 Leipzig, Germany; \\ E-Mail: mario.haim@uni-leipzig.de \\ * Corresponding author
}

Submitted: 24 June 2020 | Published: 10 July 2020

\begin{abstract}
What changes as algorithms proliferate within journalism and become more sophisticated? In this essay, we synthesize the articles in this thematic issue, which offer empirical evidence for how algorithms-and especially those designed to automate news production-are being incorporated not only into journalistic activities but also into the logics of journalism itself. They underscore that journalists have neither feared nor rejected such algorithms, as might be expected given the recent history of technological adoption in journalism. Instead, journalists have sought to normalize the technology by negotiating them against existing values and practices, and perhaps even reified some normative ideological constructs by finding unique value in what they offer as humans. These articles also highlight the shortcomings of those algorithms, giving pause to postulations of algorithms as potential solutions to shortcomings of trust in news and market failures. Indeed, such algorithms may end up amplifying the very biases that seed distrust in news all the while appearing less valuable to readers than their human counterparts. We also point to new opportunities for research, including examinations of how algorithms shape other stages in the journalistic workflow, such as interviewing sources, organizing knowledge, and verifying claims. We further point to the need to investigate higher analytic levels and incorporate additional perspectives, both from more diverse contexts (e.g., Global South) and from our sister academic fields (e.g., human-computer interaction). We conclude with optimism about the continued contributions this stream of work is poised to make in the years to come.
\end{abstract}

\section{Keywords}

actants; algorithmic journalism; algorithms; automated journalism; automation; journalism; journalism studies; robot journalism

\section{Issue}

This editorial is part of the issue "Algorithms and Journalism: Exploring (Re)Configurations" edited by Rodrigo Zamith (University of Massachusetts-Amherst, USA) and Mario Haim (University of Leipzig, Germany).

(C) 2020 by the authors; licensee Cogitatio (Lisbon, Portugal). This article is licensed under a Creative Commons Attribution 4.0 International License (CC BY).

\section{Introduction}

Journalism and technology are inseparable. While this is not a novel phenomenon, scholars have examined in recent decades the digital transformation of news, as pens gave way to keyboards, printing presses to content management systems, and postal services to internet service providers and social media (Zamith \& Braun, 2019). However, although technology has historically been an ever-present partner, it was just that: an aide to a humanled endeavor.

In some sectors of journalism, this has changed. Algorithmic actants - software that follow a prespecified set of instructions to computationally transform some input into different output-are no longer just partners (Lewis \& Westlund, 2015; Zamith, 2019). With growing independence, they now report and write stories, automatically adapt and disseminate content across multiple 
information channels, and personalize journalistic products based on information about individual news consumers (Diakopoulos, 2019; Haim \& Graefe, 2017). In these instances, it is the human that is the aide in an algorithm-led endeavor.

With this in mind, we organized this thematic issue around the idea of what changes as algorithms proliferate within journalism and become more sophisticated. How are existing activities, relationships, roles, and rewards reconfigured? To what extent do those reconfigurations require scholars to revisit existing theories and methods for understanding emerging assemblages, practices, and norms? These questions are not intended to convey technological determinism. Rather, they recognize that technology has long played a crucial role in journalism and merits special attention in today's media environments, even as technology in journalism is itself shaped by and implicated in broader questions about culture, politics, and economics (Zamith \& Braun, 2019).

The articles in this thematic issue address some of these questions but also reveal something about our current moment as a field. Specifically, they demonstrate a fascination with automation: Nearly all concern themselves with the perceptions and impacts of algorithms used to independently perform news production activities previously carried out by humans. As this extensive connection to automation was dominant among nearly all of the submissions offered, we believe this is, at least in part, a reflection of the dominant discourses about the future of journalism, which emphasize economic insecurity, computational efficiency, and audience fragmentation and empowerment. These are not outlandish beliefs, of course. Traditional business models have buckled (Chyi \& Tenenboim, 2019), we remain in an era of 'big data' and computational fetishization (Wu, Tandoc, \& Salmon, 2019), and audiences are scattered across platforms and devices and expect some degree of control over the content they consume (Engelke, 2019).

However, the articles in this thematic issue suggest that, as manifested in contemporary practice, automation-induced changes are generally modest insofar as they only apply in certain scenarios, are typically not very sophisticated, and do not significantly trouble journalists. This is not to say that journalism is immune to automation -there is ample evidence that some change has already occurred and that many more fundamental transformations are likely yet (Diakopoulos, 2019). But the discrepancy between the rhetorical and empirical does remind us of the near-term obstacles to such change within the space of journalism, from the technical challenges of replicating the art of interviewing to the cultural challenges of advancing civic-minded and human-centric pursuits.

\section{Advancing Our Understanding of Algorithms}

The contributions to this thematic issue help us better understand some of those challenges, which apply not only to the space of automation but also to broader algorithmically oriented actors and activities.

Kunert's (2020) interviews with data and software providers and media outlets in Germany's sports journalism space found that the actors involved in news production believed that automated journalism offered added value for their readers-for example, it could cover events that might otherwise be ignored-but still believed human intervention was necessary for preserving quality. Kunert further notes that automation is currently used most often to write less-important and peripheral stories, and is therefore more of a complementary tool for journalists. Thus, although automated journalism introduced new actors, actants, and activities to the space of journalism, its traditional practitioners did not believe a serious reconceptualization of their social roles as journalists was needed.

Schapals and Porlezza's (2020) interviews with German journalists outside the genre of sports echoed some of those findings. They similarly found that journalists did not feel threatened by algorithms designed to automate their work. Instead, they believed automation would free them from monotonous, day-to-day stories and allow them to focus on more meaningful, indepth stories-which they believed could not be easily automated. Moreover broadly, Schapals and Porlezza's interviewees drew upon traditional role conceptions in articulating a defense of their human contributions. Consequently, as the authors argue, automation has reified rather than replaced traditional role conceptions and thus promoted ideological continuity in the face of potential disruption.

Peterson-Salahuddin and Diakopoulos (2020) also found, through interviews with U.S. journalists, that social media algorithms were perceived to be most influential to editorial decision-making when the algorithm's indication of newsworthiness aligned with traditional understandings of newsworthiness. Put differently, journalists did not believe their editorial logics were determined by algorithmic feedback. Instead, such feedback was used in a complementary fashion, alongside traditional journalistic norms, for informing different gatekeeping decisions. This leads Peterson-Salahuddin and Diakopoulos to conclude that while social media algorithms have complicated gatekeeping practices, journalists still draw heavily upon traditional values in their decision-making.

Leppänen, Tuulonen, and Sirén-Heikel (2020) call attention to the ways in which the algorithms that power automated news production can become intentionally and unintentionally biased. They aptly illustrate why the myth of the 'mechanical objectivity' of algorithms is problematic, and advance the discussion by highlighting both the overt ways in which humans may introduce biases and the subtle ways in which actants may amplify them. Leppänen and colleagues also seed future work aimed at auditing algorithms by proposing strategies for evaluating algorithmic biases that take into account different 
levels of cooperation from the system's owners. This is important given that many such algorithms are not only proprietary but effectively 'black boxes' that are methodologically challenging to evaluate.

Graefe and Bohlken (2020) observe in a metaanalysis of ten studies comparing readers' perceptions of computer-generated and human-written news that there was no difference in perceptions of credibility, a very small preference for human-written news in terms of quality, and a sizable preference for human-written news with respect to readability. However, when explicitly told about the authorship of a story, participants generally provided higher ratings across credibility, quality, and readability if the author was a human. This pushes back against arguments that audiences perceive algorithms, and their 'mechanical objectivity,' to be superior to and more trustworthy than their human counterparts.

Collectively, these articles offer empirical evidence for how algorithms-and especially those designed to automate news production-are being incorporated not only into journalistic activities but also into the logics of journalism itself. They underscore that journalists have neither feared nor rejected such algorithms, as might be expected given the recent history of technological adoption in journalism. Instead, journalists have sought to normalize the technology by negotiating them against existing values and practices, and perhaps even reified some normative ideological constructs by finding unique value in what they offer as humans. These articles also highlight the shortcomings of those algorithms, giving pause to postulations of algorithms as potential solutions to shortcomings of trust in news and market failures. Indeed, such algorithms may end up amplifying the very biases that seed distrust in news all the while appearing less valuable to readers than their human counterparts.

\section{Opportunities for Future Work}

The growing integration of algorithmic actants into journalistic logics opens up new pathways for evaluating their interdependency with human actors and the potential biases they mitigate and reinforce. In light of an increasingly institutionalized relationship, the design, development, and deployment of algorithms not only potentially affects journalistic processes and proclivities but may actively shape everyday coverage, too. However, while algorithmic actants have been shown to impact newswriting, news distribution, and audience perceptions, far less is known about how they shape other stages in the journalistic workflow, such as interviewing sources, organizing knowledge, and verifying claims. Those areas strongly merit further scholarly attention.

Moreover, much of the literature to date focuses on case studies and micro-level comparative analyses. These offer essential steps in theorizing algorithmic actants within journalism studies. However, in order to continue to move this stream of work forward, it is nec- essary to investigate higher levels and additional perspectives-certainly those beyond North America and Europe. This opens room to investigate the extent to which distinct journalism cultures moderate and accentuate certain beliefs and practices, and further how new organizational structures and economic models may be emerging alongside and in response to algorithmically enabled affordances.

Finally, much of the work in this space to date has been qualitative or conceptual in nature, aiming to describe and make some sense of these developments. These works have been essential to developing our understanding, but they also run the risk of becoming limited by methodological and theoretical homogeneity. To that end, we encourage scholars to consider additional theoretical perspectives-including those from our sister fields of human-computer interaction and organizational studies-as well as computational methodologies that aim to capture nuances about what and how algorithms perform as they are deployed within journalism. Ultimately, we remain very optimistic about the continued contributions this stream of work is poised to make in the years to come.

\section{Acknowledgments}

We wish to thank the many reviewers who offered prompt, thoughtful, and thorough evaluations of the papers considered for this thematic issue. We also wish to thank the authors, all of whom worked resolutely to meaningfully engage with the reviewers' comments and repeatedly produce stronger revisions. Finally, we wish to thank the editorial team at Media and Communication, who made it impossible for us to miss our deadlines with their expediency and professionalism.

\section{Conflict of Interests}

The authors declare no conflict of interests.

\section{References}

Chyi, H. I., \& Tenenboim, O. (2019). From analog dollars to digital dimes: A look into the performance of US newspapers. Journalism Practice, 13(8), 988-992. https://doi.org/10.1080/17512786.2019.1642793

Diakopoulos, N. (2019). Automating the news: How algorithms are rewriting the media. Cambridge, MA: Harvard University Press.

Engelke, K. M. (2019). Online participatory journalism: A systematic literature review. Media and Communication, 7(4), 31-44. https://doi.org/10.17645/mac. v7i4. 2250

Graefe, A., \& Bohlken, N. (2020). Automated journalism: A meta-analysis of readers' perceptions of humanwritten in comparison to automated news. Media and Communication, 8(3), 50-59.

Haim, M., \& Graefe, A. (2017). Automated news. Dig- 
ital Journalism, 5(8), 1044-1059. https://doi.org/ 10.1080/21670811.2017.1345643

Kunert, J. (2020). Automation in sports reporting: Strategies of data providers, software providers, and media outlets. Media and Communication, 8(3), 5-15.

Leppänen, L., Tuulonen, H., \& Sirén-Heikel, S. (2020). Automated journalism as a source of and a diagnostic device for bias in reporting. Media and Communication, 8(3), 39-49.

Lewis, S. C., \& Westlund, O. (2015). Actors, actants, audiences, and activities in cross-media news work. Digital Journalism, 3(1), 19-37. https://doi.org/10.1080/ 21670811.2014.927986

Peterson-Salahuddin, C., \& Diakopoulos, N. (2020). Negotiated autonomy: The role of social media algorithms in editorial decision making. Media and Communication, 8(3), 27-38.

Schapals, A. K., \& Porlezza, C. (2020). Assistance or resistance? Evaluating the intersection of automated jour- nalism and journalistic role conceptions. Media and Communication, 8(3), 16-26.

Wu, S., Tandoc, E. C., \& Salmon, C. T. (2019). When journalism and automation intersect: Assessing the influence of the technological field on contemporary newsrooms. Journalism Practice, 13(10), 1238-1254. https://doi.org/10.1080/17512786.2019.1585198

Zamith, R. (2019). Algorithms and journalism. In H. Örnebring, Y. Y. Chan, M. Carlson, S. Craft, M. Karlsson, H. Sjøvaag, \& H. Wasserman (Eds.), Oxford encyclopedia of journalism studies (pp. 1-21). Oxford: Oxford University Press. https://doi.org/10.1093/acrefore/ 9780190228613.013.779

Zamith, R., \& Braun, J. A. (2019). Technology and journalism. In T. P. Vos, F. Hanusch, D. Dimitrakopoulou, M. Geertsema-Sligh, \& A. Sehl (Eds.), The international encyclopedia of journalism studies (pp. 1-7). Hoboken, NJ: Wiley. https://doi.org/10.1002/ 9781118841570.iejs0040

\section{About the Authors}

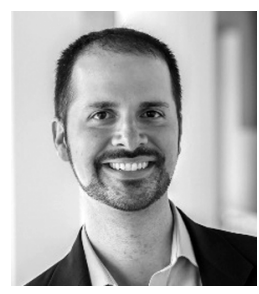

Rodrigo Zamith (PhD, University of Minnesota) is an Associate Professor in the Journalism Department at the University of Massachusetts Amherst and a Faculty Associate at UMass' Computational Social Science Institute. His research interests lie at the intersection of journalism and technology, with a focus on the reconfiguration of journalism in a changing media environment and the development of digital research methods for social scientists. Zamith's most recent work can be found at https://www.rodrigozamith.com.

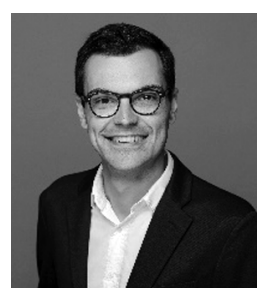

Mario Haim (PhD, LMU Munich) is a Junior Professor at the Department of Communication and Media Studies at the University of Leipzig, Germany. His research interests include algorithmic influences on journalism, such as within automated and data-driven journalism as well as through audience metrics, the use of news within algorithmically curated media environments, and the technological means of online health communication. He also has a keen interest in computational methods. More information can be found at https://www.haim.it. 